\title{
Aplicación del Modelo de Interacción lónica de Pitzer para determinar Coeficientes de Actividad en Salmueras Naturales con Presencia de Boro
}

\author{
Ingrid Garcés ${ }^{1}$ \\ (1) Universidad de Antofagasta, Dpto. de Ing. Química y Procesos Minerales, Casilla 170, Antofagasta-Chile \\ (e-mail: ingrid.garces@uantof.cl)
}

Recibido Jul. 10, 2018; Aceptado Sep. 25, 2018; Versión final Nov. 1, 2018, Publicado Abr. 2019

\begin{abstract}
Resumen
Se aplica el modelo de interacción iónico desarrollado por Pitzer a las salmueras naturales del Salar de Loyoques. Este salar está ubicado en el Altiplano de la Región de Antofagasta-Chile, a 4430 metros sobre el nivel del mar. La principal característica del salar es que presenta en su centro una boratera con niveles explotables de ulexita, un borato doble de sodio y calcio. Las solubilidades de los minerales en lagos salinos y coeficientes de actividad se pueden calcular a partir de consideraciones termodinámicas, haciendo uso del código de especiación PHRQPITZ. En este contexto, el objetivo del trabajo fue evaluar los coeficientes de actividad y la secuencia mineralógica de las salmueras de dicho salar. Los resultados indican que las aguas del salar de Loyoques muestran una considerable variedad tanto en la composición iónica, como en su concentración, son aguas levemente alcalinas y su clasificación es del tipo $\mathrm{Na}-\mathrm{Cl}-\mathrm{SO}_{4}$. Las aguas que ingresan a esta cuenca cerrada, bajo un clima árido con volcanismo activo, se someten a la concentración por evaporación dando lugar a precipitación de calcita y presentando sólo algunas muestras próximas al equilibrio con yeso y magnesita.
\end{abstract}

Palabras clave: coeficientes de actividad; boratos; depósitos andinos; modelo de Pitzer; Salar de Loyoques; PHRQPITZ

\section{Application of Pitzer ion interaction model in the determination of activity coefficient to natural brines with presence of Boron}

\begin{abstract}
The ionic interaction model developed by Pitzer is applied to determine activity coefficient of the evaporitic Andean deposits known as Salar de Loyoques, in northern Chile. This salar is located in the Altiplano zone of the region of Antofagasta-Chile, at $\mathbf{4 4 3 0}$ meters above sea level. The main characteristic of the salar is that in its center it has a borax deposit with exploitable levels of ulexita, a double borate of sodium and calcium. The solubilities of the minerals and the activity coefficients were calculated based on thermodynamics fundamentals using the software PHRQPITZ. In this context, the objective of this work was to evaluate the activity coefficients and the mineralogical sequence of the salar brines. The results indicate that the waters of the Loyoques salar's show considerable variations both in their composition and their concentration but are slightly alkaline, and belong to the type $\mathrm{Na}-\mathrm{Cl}-\mathrm{SO}_{4}$. All the waters that enter this closed basin, subjected to processes of concentration by evaporation which causes the precipitation of calcite and later the samples that are closer to balance will be the gypsum and magnesite.
\end{abstract}

Keywords: activity coefficients; borates; andean deposits; Pitzer's model; Loyoques salar's; PHRQPITZ 


\section{INTRODUCCION}

El interés que presenta el estudio de sistemas salinos se basa en la obtención de datos que ayuden a comprender los procesos activos que tienen lugar en estos medios, como también su evolución hidrológica y geoquímica que dependen fuertemente de la evaporación del agua y la precipitación mineralógica de estas cuencas. Estudios geoquímicos en sistemas salinos desarrollados en ambientes continentales tienen asociado varios inconvenientes, entre los que se pueden destacar, los efectos causados por los diferentes tipos de procesos, como por ejemplo, cuando se modifica la concentración de solución por causa de un determinado elemento producto de la evaporación o bien por la variación de temperatura que ocurre en el medio (Auqué et al.,1995); otra dificultad es el seguimiento de las etapas finales en las que tiene lugar la evaporación de las salmueras, debido a la restricción espacial y a los problemas asociados en el muestreo de las soluciones residuales; $y$, finalmente, enumerar las dificultades que tiene el estudio fisicoquímico de estos sistemas, por la alta concentración de las salmueras lo que limita la aplicabilidad de los códigos de modelización geoquímica basados en los clásicos planteamientos de cálculo de coeficientes de actividad (López et al., 1999). La resolución de todos estos problemas precisa la combinación de un conocimiento detallado del sistema natural con la información suministrada por el estudio fisicoquímico del mismo (Weare, 1987). La utilización de esta metodología en el estudio de un sistema salino continental permite analizar la evolución de las salmueras sometidas a un proceso de concentración por evaporación, y también obtener la secuencia de cristalización de sales que se produce bajo esas condiciones. La descripción de composiciones iónicas y sus cambios temporales en los cuerpos de agua naturales cerrados permiten dar explicación del mecanismo de formación y el desarrollo de enriquecimiento de sus salmueras. La salinidad de las aguas puede provenir de la alteración de rocas volcánicas y sedimentarias, o de la redisolución de antiguas evaporitas, o bien de la oxidación del azufre y de minerales sulfurados, o de cuñas salinas.

En este artículo se presentan los resultados del tratamiento fisicoquímico llevado a cabo a partir de las salmueras naturales del Salar de Loyoques, ubicado en la alta cordillera al noreste de Chile (Figura 1), cuyas características hidroquímica se conocen gracias a los estudios de Risacher et al. (1999). Las observaciones realizadas por estos autores sobre el propio sistema natural han servido como referencia a la hora de interpretar los resultados ofrecidos de la modelización geoquímica.

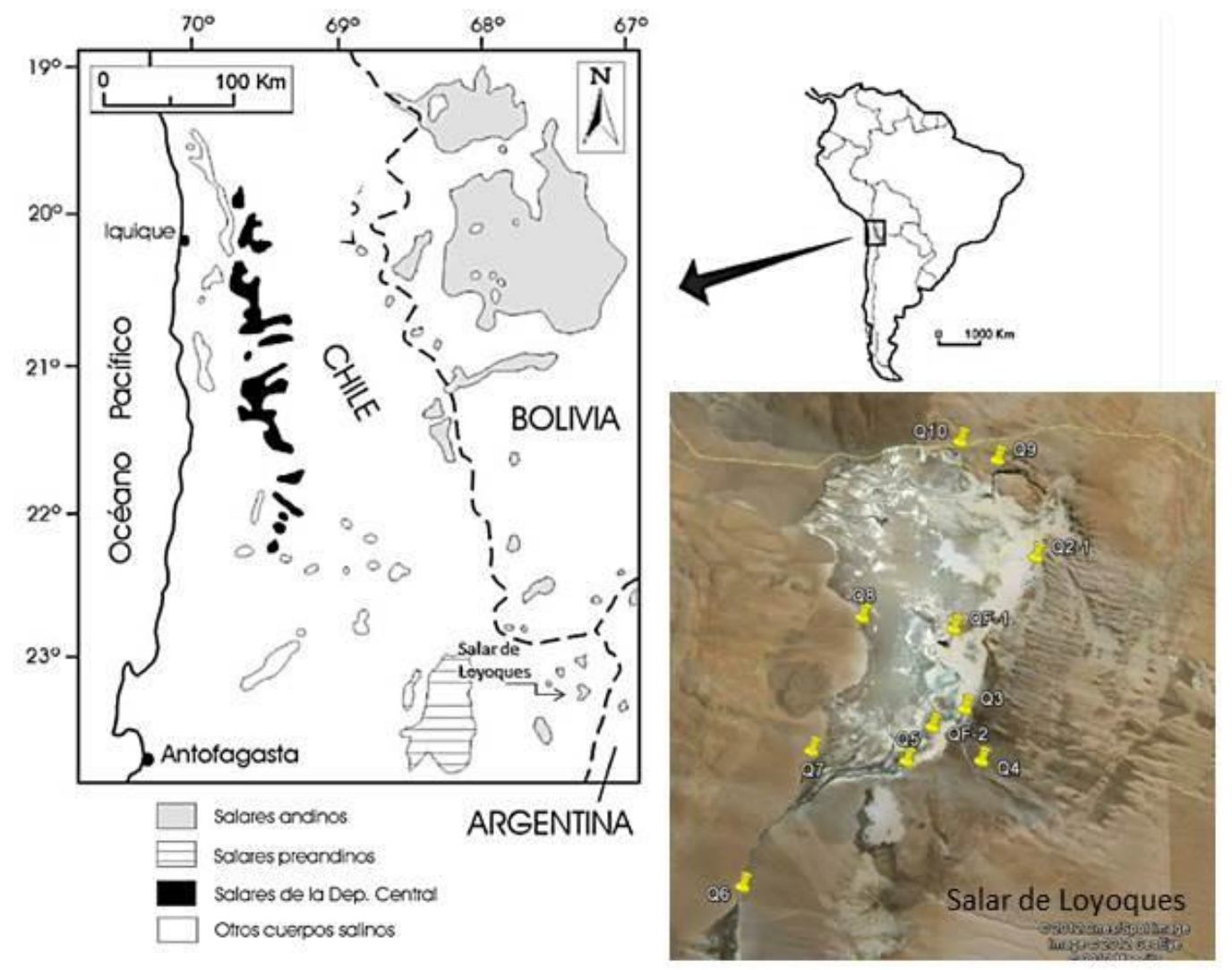

Fig. 1: Ubicación geográfica del Salar de Loyoques, con representación en el extremo inferior derecho, los puntos de muestreos de aguas efectuados en el Salar de Loyoques.

Los salares con boratos en los Andes Centrales alcanzan a un medio centenar de depósitos con mineralización de ulexita y excepcionalmente tincal. Perú cuenta con un único yacimiento (Laguna Salinas, Arequipa), donde además de ulexita ocurre inyoita. Mientras que, Bolivia tiene depósitos de ulexita en los 
salares de Coipasa, Empexa, Uyuni, Chiguana, Pastos Grandes, Capina, Mamacoma, Curuto, Chalviri, Luriques y otros. Chile también posee salares andinos con reservas de ulexita. Los depósitos más importantes son los de Surire, Ascotán, Quisquiro y Carcote. En Argentina los principales salares boratíferos son Hombre Muerto, Diablillos, Ratones, Centenario, Cauchari, Olaroz y Salinas Grandes. Los boratos son sales solubles fácilmente meteorizables y desaparecen de la superficie (Alonso, 2012).

El clima de la región es bastante variable, específicamente en el área en que se encuentra el salar. Los diferentes tipos de clima y el efecto del cambio climático es uno de los principales factores para comprender la evolución de sus aguas, y en el caso del área geográfica en que se encuentran los depósitos andinos, una componente esencial es la altitud. Recientemente, Sarricolea et al., (2017) actualizaron datos y mapa, proponiendo una nueva clasificación regional en que se considera el cambio climático. La figura 2 muestra el mapa con las actuales zonas climáticas de Chile, basado en la clasificación de Köppen-Geiger, con las superficies bioclimáticas de Pliscoff, Luebert, Hilger y Guisan (2014) e incorpora los datos del período 1950 2000. La efectividad del sistema de clasificación Köppen-Geiger se trata ampliamente en Peel et al. (2007).

La clasificación climática de Köppen-Geiger en su estructura es de tercer orden. El primer criterio considera los cinco tipos de clima que siguen las bandas latitudinales de la temperatura y la disponibilidad de agua: A (tropical), B (árido), C (templado), D (frío) y E (polar). El segundo criterio climático es de naturaleza pluviométrica. Los climas tropicales incluyen Af (precipitación del mes más seco por encima de $60 \mathrm{~mm}$ ), Am (para la sequía monzónica) y Aw (para climas de sabana). Para climas $C$ y $D$, el segundo orden indica una temporada seca de verano con una 's' (Cs y Ds), 'W' en invierno (Cw y Dw), y 'f' para climas sin una estación seca (Cf y Df). Para climas B y E, el segundo orden está en mayúscula (W, S, T y F) y clasifica climas en el desierto (BW), semiárido (BS), tundra (ET) y capa de hielo (EF). El tercer orden es para criterios térmicos, se usa en climas B, C y D; el clima B puede ser 'h' (cálido) (BWh y BSh) o 'k' (frío) (BWk y BSk); para climas templados, el tercer orden indica condiciones térmicas que son calientes (a), cálidas (b) o leve (c); y para climas $D$, el tercer orden incluye a, b y c, así como una variante fría (d). El área en que se ubica el salar es bastante variable y corresponde al tipo polar, clima Tundra, cuya principal característica son inviernos secos.

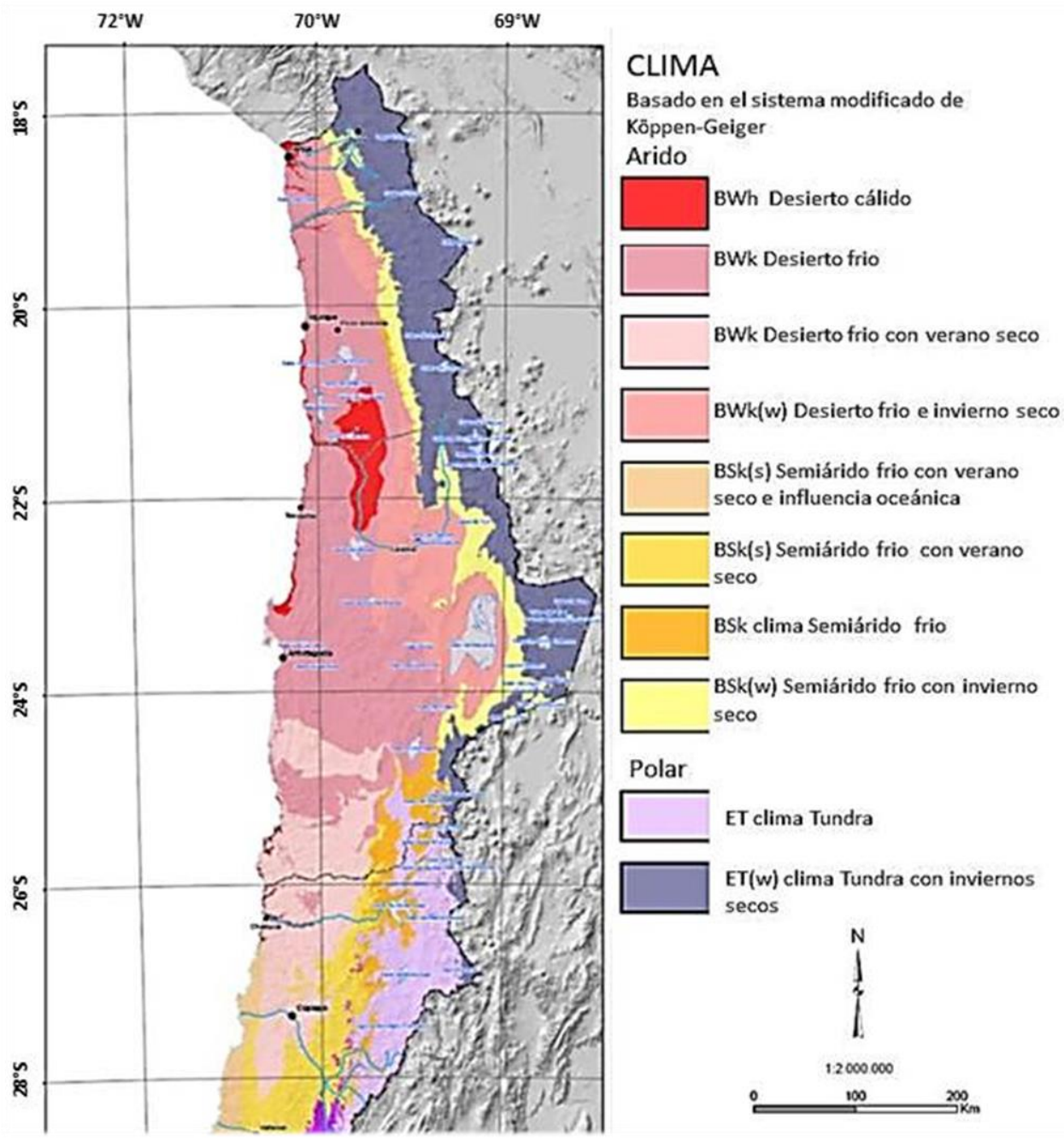

Fig. 2: Climas de la región del norte de Chile (Tomado y modificado de Sarricolea et al., 2017). 


\section{AREA DE ESTUDIO}

El Salar de Loyoques (o Quisquiro) se ubica en el Altiplano de la región de Antofagasta (Fig. 1) entre las latitudes $23^{\circ} 07^{\prime}$ y $23^{\circ} 30^{\prime}$ latitud sur y las longitudes $67^{\circ} 10^{\prime}$ y 67응'oeste, a 4.430 m.s.n.m. Este depósito salino es una cuenca cerrada de unos $80 \mathrm{~km}^{2}$, en la que convergen las aguas caídas sobre su hoya hidrográfica que alcanza unos $749 \mathrm{~km}^{2}$. Es un salar tipo playa con pocas lagunas superficiales de extensión variable (alrededor de $5 \mathrm{~km}^{2}$ ), una napa de salmuera de unos decímetros de profundidad, cuya principal característica es presentar en su centro una boratera con niveles explotables de ulexita, un borato doble de sodio y calcio (Garcés y López, 2012). Para que se forme un yacimiento de boratos es necesaria la presencia de una cuenca cerrada que pueda albergar un cuerpo de agua somero, sometido a evaporación en una región árida con actividad volcánica reciente y fuentes termales portadoras del elemento boro.

Los principales aportes superficiales al salar son el río Salado por el sur, el cual nace en las vegas Ojos del Salado y por el norte el estero Loyoques, además de tres quebradas por el poniente (de norte a sur) Quebrada de Agua Escondida, Quebrada de Taina y Quebrada de Quisquiro. La evaporación superficial de las aguas desde el suelo es muy alta, varía entre 1.500 a $2.500 \mathrm{~mm} /$ año (Risacher et al., 1999). La precipitación anual media a los $4.000 \mathrm{msnm}$, alcanza entre 200 a $250 \mathrm{~mm}$, lo cual explica que al comparar con la evaporación, se tiene una alta aridez. Los años lluviosos pueden tener hasta tres veces las precipitaciones de un año seco. En el caso específico del Salar de Loyoques, la máxima temperatura media diaria oscila entre $10^{\circ}$ y $20^{\circ} \mathrm{C}$, mientras que, la temperatura media mínima está a unos pocos grados bajo 0 (D.G.A., 1987). Los vientos en el altiplano siguen un patrón, que por lo general es en las mañanas de escasa intensidad con orientación norte, mientras que por las tardes comienzan de moderada a alta intensidad, con orientación oeste a este.

\section{MODELIZACIÓN GEOQUÍMICA}

El modelado se basa en la aplicación de principios fisicoquímicos al estudio e interpretación de sistemas hidrogeoquímicos, y su uso actual abarca prácticamente todos los campos donde existen interacciones aguaroca (hidroquímica superficial y subterránea, hidrotermalismo, edafología). En procesos de separación de salmueras naturales, las sales que cristalizan en un determinado sistema, requieren del conocimiento del equilibrio sólido-líquido de dicho sistema, lo que a su vez requiere del conocimiento de los coeficientes de actividad de los electrolitos presentes en dichas salmueras. El código utilizado para los cálculos de coeficientes de actividades de las salmueras estudiadas, es el PHRQPITZ (Plummer et al., 1988), al que se le incorpora y amplía a rangos de temperatura de $0^{\circ}$ a $60^{\circ} \mathrm{C}$ la aproximación de Pitzer y la parametrización realizada por Harvie y colaboradores (1984) para el sistema Na-K-Mg-Ca-H-Cl-SO $4-\mathrm{OH}-\mathrm{HCO}_{3}-\mathrm{CO}_{3}-\mathrm{CO}_{2}-\mathrm{H}_{2} \mathrm{O}$, aplicable en un principio sólo a $2.5^{\circ} \mathrm{C}$. Los códigos están sujetos al cumplimiento de condiciones de equilibrio químico y de conservación de masa y/o de carga, lo que se traduce en una serie de ecuaciones de gobierno en que resulta fundamental el conocimiento de las actividades de las especies presentes. Este software calcula las actividades iónicas individuales a partir de concentraciones analíticas, basado en el Método de Interacción Específica. Posteriormente, evalúa el grado de saturación de las soluciones respecto a las fases minerales de interés y compara los productos obtenidos de actividad iónica (P.A.I.), frente a las constantes de equilibrio (Keq) del mineral correspondiente, por medio del índice de saturación (I.S.):

$$
\text { I.S. }=\log (\text { P.A.I./ Keq) }
$$

En esta ecuación, P.A.I. representa el producto de actividad iónica y Keq la constante de equilibrio correspondiente. Si el índice de saturación muestra valor cero significa que la solución está en equilibrio respecto a esa fase; por otra parte, valores positivos o negativos del I.S. determinan una situación de sobresaturación o subsaturación, respectivamente, cuya magnitud es función directa del valor absoluto de dicha cantidad. La solubilidad de un mineral en el equilibrio puede ser representado como:

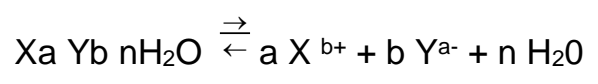

Si el producto de solubilidad de un mineral se define como $\left(\mathrm{Ksp}, \mathrm{XaYb} \bullet \mathrm{nH}_{2} \mathrm{O}\right)$ y se considera como la actividad en fase sólida igual a 1 , entonces

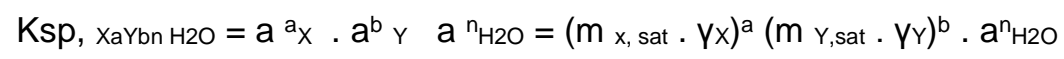

Donde $\mathrm{m} x$,sat y $\mathrm{m} \mathrm{Y}$,sat son las concentraciones molales del catión y anión respectivamente, en la fase líquida saturada con respecto a la fase sólida. Por ejemplo, para el yeso la ecuación 2 tomará la forma:

$$
\mathrm{Ca}_{2} \mathrm{SO}_{4} 2 \mathrm{H}_{2} \mathrm{O} \stackrel{\rightarrow}{\leftarrow} \mathrm{Ca}^{+2}+\mathrm{SO}_{4}^{-2}+2 \mathrm{H}_{2} \mathrm{O}
$$


El producto de solubilidad del yeso $\left(\mathrm{Kps}, \mathrm{Ca}_{2} \mathrm{SO}_{4} \cdot 2 \mathrm{H}_{2} \mathrm{O}\right)$ en función de la actividad queda como:

$$
\mathrm{Kps}=\left(\mathrm{a} \mathrm{Ca++} \cdot \mathrm{a} \text { SO4-- } \cdot \mathrm{a}^{2} \mathrm{H} 2 \mathrm{O}\right) / \mathrm{a} \mathrm{CaSO}_{2} \mathrm{H} 2 \mathrm{O}
$$

La actividad de la fase sólida (mineral) definida como igual a $1\left(\mathrm{aCaSO}_{4} \cdot 2 \mathrm{H}_{2} \mathrm{O}=1\right)$, donde la ecuación 5 se transforma en:

$$
\mathrm{Kps}=\left(\mathrm{m} \mathrm{Ca++} \cdot \mathrm{YCa++}_{+}\right)\left(\mathrm{msO4--}_{\mathrm{SSO}--}\right) \cdot \mathrm{a}^{2} \mathrm{H} 2 \mathrm{O}
$$

Esta ecuación puede ser reescrita como:

$$
\mathrm{Kps}=\mathrm{Kps}^{*} \cdot \mathrm{YCa++} \cdot \mathrm{YsO4--}
$$

Donde $\mathrm{Kps}^{*}$ es el producto de solubilidad estequiométrico, $\mathrm{Kps}^{*}=\left(\mathrm{m} \mathrm{Ca++}{ }^{*} \mathrm{msO4}_{\mathrm{s}-}\right)$

Es así como en el equilibrio, el producto de solubilidad depende del producto termodinámico y estequiométrico. El coeficiente de actividad $(\mathrm{V})$ y la solubilidad dependen de varios factores, entre el principal está la fuerza iónica (I). Ahora bien, como los coeficientes de actividad disminuyen con la fuerza iónica del medio, el valor de Kps deberá aumentar en función de la fuerza iónica (I), dado que la constante de solubilidad termodinámica no varía. Este aumento de la constante estequiométrica (Kps) se traduce en un aumento de la solubilidad, como se deduce de la ecuación 7 , y que se conoce con el nombre de efecto salino. La evaluación del estado de saturación de las soluciones frente a las fases minerales seleccionadas se ha llevado a cabo calculando los índices de saturación. Las constantes de equilibrio empleadas en los cálculos de saturación son las que aparecen en la base de datos del código utilizado.

El modelo de Pitzer se puede considerar como una extensión del Modelo Debye-Hückel. La formulación general del modelo de Pitzer puede ser descrito por la ecuación. (8) que presenta el exceso energía total de Gibbs. La primera parte de esta ecuación corresponde a la Modelo Debye-Hückel y es una función de la fuerza iónica y la constante dieléctrica del disolvente (Hamrouni y Dhahbi, 2001). El segundo y el tercer término se introducen para modelar las interacciones binarias y las ternarias que no fueron citadas en el Modelo DebyeHückel inicial. Pitzer presentó y formuló estos dos términos para describir el comportamiento de las soluciones en altas concentraciones (Hamrouni y Dhahbi, 2001).

$$
\mathrm{Gex} /(\mathrm{nw} \mathrm{Rg} T)=\mathrm{f}(\mathrm{I})+\Sigma_{\mathrm{i}} \Sigma_{\mathrm{j}} \lambda_{\mathrm{ij}} \mathrm{m}_{\mathrm{i}} \mathrm{m}_{\mathrm{j}}+\Sigma_{\mathrm{i}} \Sigma_{\mathrm{j}} \sum_{\mathrm{k}} \mu_{\mathrm{ijk}} \mathrm{m}_{\mathrm{i}} \mathrm{m}_{\mathrm{j}} \mathrm{m}_{\mathrm{k}}
$$

donde $f(I)$ es una función de la fuerza iónica, que expresa el efecto de la fuerzas electrostáticas de largo alcance; i, j y k son aniones diferentes (o cationes), $m_{i}$ denota la molalidad del i-ésimo ion (moles por kilogramo) y nw es el número de kilogramos de agua. $\lambda_{\mathrm{ij}}$ es un segundo coeficiente virial que expresa el efecto de las fuerzas de corto alcance entre especies i y j. $\lambda_{\mathrm{ij}}$ depende de la fuerza iónica. $\mu_{\mathrm{ijk}}$ es el tercer coeficiente virial que explica las interacciones de triples iones; $\mu_{\mathrm{ijk}}$ es independiente de la fuerza iónica (Pitzer y Kim, 1974). Las ecuaciones del coeficiente de actividad se obtienen por medio de derivaciones de ecuación (8), mayor detalle descrito en Pitzer y Mayorga (1973), Pitzer (1975, 1991) y Pitzer y Kim (1974).

\section{METODOLOGIA EXPERIMENTAL}

En terreno se tomaron muestras de aguas, y se les midió la temperatura, $\mathrm{pH}$, salinidad y conductividad eléctrica. El pHmetro y la conductancia se calibraron de forma periódica con soluciones tampón. Las aguas colectadas en el salar se recogieron en botellas de polietileno de 1 litro, previamente rotuladas y ubicadas geográficamente con GPS. La Tabla 1, resume los resultados de los análisis realizados a las muestras de aguas tomadas durante dos campañas, enero y noviembre, en los mismos puntos de extracción. Durante el transporte al laboratorio, las muestras se mantuvieron refrigeradas, al resguardo de la luz y selladas. En el laboratorio se determinaron los cationes (K, Na, Ca, Mg, Li y As) por espectrofotometría de absorción atómica (Perkin Elmer 2380), utilizando el protocolo estándar. Los cloruros por el método de Mohr, $\mathrm{SO}_{4}$ por gravimetría con $\mathrm{BaCl}$ y análisis volumétrico $\left(\mathrm{Cl}, \mathrm{B}_{2} \mathrm{O}_{3}, \mathrm{CO}_{3}, \mathrm{HCO}_{3}\right)$. La Tabla 1 muestra los resultados de los análisis. La calidad de los datos analíticos se evalúa mediante el cálculo del porcentaje de error de balance de cargas, por la expresión de Ball y Nordstrom (1991). Paralelamente, se toman muestras de costras salinas y sedimentos, las que una vez secas, se analiza su mineralogía con un difractómetro de Absorción Atómica (Siemens, modelo D5000).

Se utiliza un modelo geoquímico como metodología complementaria para los datos de campo y analíticos de soluciones y sedimentos naturales. Estos proporcionan resultados para la interpretación de los procesos de precipitación y disolución de minerales en medios salinos (Krumgalz, 2001). Se utilizó el código de modelización geoquímico PHRQPITZ (Plummer et al., 1988), con el fin de evaluar las actividades iónicas e 
índices de saturación, los que darán cuenta de la secuencia de minerales que controlan la evolución geoquímica de las salmueras. La Tabla 2 muestra los resultados de los índices de saturación de los principales minerales posibles de precipitar, los que son calculados por el programa mediante la ecuación 1. Si el índice de saturación (I.S.) muestra valor cero significa que la solución está en equilibrio respecto a esa fase. Valores positivos o negativos del IS, indican una situación de sobresaturación o subsaturación, respectivamente, cuya magnitud es función directa del valor absoluto de dicha cantidad.

Tabla 1: Composición química en meq/l de las salmueras del Salar de Loyoques. El término n.d. es no determinado.

\begin{tabular}{|c|c|c|c|c|c|c|c|c|c|c|c|}
\hline Muestra & $\mathrm{Li}$ & $\mathrm{K}$ & $\mathrm{Na}$ & $\mathrm{Ca}$ & $\mathrm{Mg}$ & $\mathrm{Cl}$ & $\mathrm{SO}_{4}$ & $\mathrm{HCO}_{3}$ & $\mathrm{~B}_{2} \mathrm{O}_{3}$ & $\mathrm{As}$ & $F$ \\
\hline Q3E & 0.10 & 0.87 & 13.70 & 6.34 & $0 . .91$ & 6.49 & 11.40 & 3.77 & 9.25 & 10.06 & 0.09 \\
\hline Q4E & 0.01 & 0.15 & 1.39 & 1.048 & 0.13 & 0.66 & 1.38 & 0.87 & 4.40 & 6.54 & 0.02 \\
\hline Q5E & 1.37 & 11.25 & 120.8 & 14.72 & 10.95 & 124.3 & 84.25 & 2.34 & 14.91 & 163.84 & 0.50 \\
\hline Q6E & 0.49 & 1.71 & 74.51 & 15.17 & 5.02 & 87.79 & 5.77 & 1.12 & 4.71 & 9.05 & 0.09 \\
\hline Q7E & 0.35 & 0.72 & 39.10 & 12.13 & 2.22 & 49.73 & 4.04 & 0.74 & 4.39 & 6.78 & 0.05 \\
\hline Q8E & 0.43 & 0.72 & 38.49 & 9.83 & 1.73 & 47.56 & 2.92 & 0.74 & 2.78 & 5.55 & 0.03 \\
\hline Q9E & 1.50 & 2.45 & 105.48 & 39.82 & 7.32 & 137.6 & 8.92 & 2.18 & 9.34 & 16.40 & 0.03 \\
\hline Q10E & 0.57 & 0.97 & 36.36 & 12.72 & 3.21 & 48.24 & 13.54 & 2.18 & 4.16 & 0.293 & 0.03 \\
\hline Q1-1N & n.d. & 7.62 & 280.43 & 115.45 & 14.40 & 448.29 & 25.29 & 1.33 & 3.56 & 0.023 & 0.07 \\
\hline QU-2N & n.d. & 1.56 & 81.30 & 1.40 & 3.42 & 91.62 & 5.35 & $n . d$. & n.d. & 0.01 & 0.04 \\
\hline Q2-1N & n.d. & 37.67 & 2773.9 & 265.65 & 65.84 & 3042.6 & 52.65 & 4.67 & 21,7 & 0.19 & 0.43 \\
\hline Q3N & n.d. & 0.24 & 4.96 & 1.80 & 0.15 & 1.89 & 4.17 & 1.41 & 0,19 & 0.02 & 0.07 \\
\hline Q4N & n.d. & 0.102 & 1.74 & 0.90 & 0.05 & 0.82 & 1.46 & 0.39 & 0,14 & 0.02 & 0.02 \\
\hline Q5N & n.d. & 14.12 & 197.43 & 30.5 & 8.49 & 163.55 & 94.83 & 1.92 & 2.20 & 0.24 & 0.1 \\
\hline Q6N & n.d. & 0.64 & 50.82 & 9.25 & 1.81 & 60.11 & 3.44 & 5.20 & 0.47 & 0.01 & 0.73 \\
\hline Q7N & n.d. & 0.36 & 36.15 & 9.5 & 2.18 & 44.74 & 2.27 & 0.33 & 0.50 & 0.02 & 0.03 \\
\hline Q8N & n.d. & 0.36 & 37.65 & 8.95 & 1.67 & 35.66 & 2.63 & 0.39 & 0.46 & 0.01 & 0.03 \\
\hline Q9N & n.d. & 1.89 & 81.64 & 22.71 & 4.58 & 100.5 & 9.17 & 0.64 & 7.68 & 0.01 & 0.03 \\
\hline Q10N & n.d. & 9.03 & 474.52 & 35.70 & 32 & 592.16 & 10.33 & 1.25 & 28.67 & 0.007 & 0.05 \\
\hline LO-2N & n.d. & 79.92 & 4451.3 & 403 & 255.6 & 5513.88 & 11.06 & 11.27 & 349.21 & 0.15 & 0.07 \\
\hline
\end{tabular}

\section{RESULTADOS Y DISCUSIÓN}

La tabla 1 presenta la composición química de las muestras de aguas tomadas en el salar, durante las dos campañas de muestreos. En conjunto, son soluciones con una amplia variabilidad de iones que en su mayoría tienen altos contenidos de $\mathrm{Na}$ y $\mathrm{K}$. La fuerza iónica (I), calculada a partir de los datos analíticos de estas aguas está comprendida entre $0.004 \mathrm{~m}$ a $3.65 \mathrm{~m}$. Esta variable es relevante para comprender el enriquecimiento salino que ha lugar en estos depósitos. La descripción de composiciones iónicas y sus cambios temporales en los cuerpos de agua naturales cerrados permiten dar explicación del mecanismo de formación y el desarrollo de enriquecimiento de sus salmueras. La muestra Q2-1N, tiene el aporte más salino $(\mathrm{I}=3,65 \mathrm{~m})$, y se presenta en el borde este sector norte donde predominan las vertientes diluidas. Esta agua es una vertiente difusa que muy probablemente se ha concentrado por otras soluciones salinas. Mientras Q$3 \mathrm{~N}$ y Q-4N, corresponden a los aportes más diluidos y se presenta por el borde este sector medio sur. Estas aguas de aporte diluidos no han redisuelto ningún tipo de antiguas evaporitas, por lo que probablemente deban su composición a la alteración de rocas volcánicas de la cuenca de drenaje. El flujo de agua que ingresa a esta cuenca cerrada, muestra una considerable variabilidad, tanto en la composición iónica, como en su concentración por evaporación, dando lugar a la precipitación de una secuencia de minerales, cada uno selectivamente, y por lo tanto se altera su composición. Este proceso se ve favorecido por cuanto el clima tiene como principal característica ser de inviernos secos (Fig.2). La química de la salmuera final, depende principalmente de la composición inicial del agua de flujo de entrada y de la secuencia y cantidad de minerales que precipiten en las sucesivas etapas de la evolución del agua.

La presencia de Boro en pequeñas cantidades es esencial para el crecimiento de las plantas, pero en concentraciones excesivas, pasa a ser toxica. La muestra Q2-1N tiene un alto contenido en B2O3 de $21.7 \mathrm{meq} / \mathrm{l}(1510 \mathrm{mg} / \mathrm{l})$, y la más diluida, Q4, con un contenido de $0.14 \mathrm{meq} / \mathrm{l}(10 \mathrm{mg} / \mathrm{l})$, ambas al ser 
comparadas con la Norma chilena de agua de riego (NCh.1333) que es $0.75 \mathrm{mg} / \mathrm{l}$, resultan excesivas, es decir, todas las aguas muestreadas del salar acusan altos contenidos en Boro por sobre la norma, inclusive la de FAO que considera un contenido normal entre 0 y 2 mg/l (Instituto Nacional de Normalización, 1978).

La variabilidad de la composición iónica, de las aguas del Salar de Loyoques se representa en el diagrama de la Fig. 3. Los iones dominantes son $\mathrm{Na}^{+}>\mathrm{SO}_{4}^{-2}>\mathrm{Cl}^{-}$y en menor relación $\mathrm{K}+>\mathrm{HCO}_{3}{ }^{-}>\mathrm{Ca}^{+2}>\mathrm{Mg}^{+2}>\mathrm{CO}_{3}{ }^{-}$ 2. Los puntos en el diagrama claramente indican predominio del $\mathrm{Na}$ frente al $\mathrm{Ca}$, con una baja incidencia del Mg en que no supera el 10\%. Las muestras presentan mayor variabilidad en cuanto a los aniones, existiendo un sector clorurado y otras sulfatadas-cloruradas. La presencia de $\mathrm{HCO}_{3}{ }_{3}$ se manifiesta sólo en las muestras Q3 y Q4 con más del 10\%, y de igual forma se manifiestan tanto en verano (Tabla 1, muestras terminadas en E), como en primavera (muestras terminadas en N). De lo observado en la Fig. 3, se puede concluir que existe una elevada variabilidad de tipos químicos en las soluciones superficiales del salar.

Para el cálculo de las condiciones físico-químicas se utilizó el código PHRQPITZ que permite calcular los coeficientes de actividad iónicos (vi) y el grado de saturación de las aguas. La Fig. 4 muestra la evolución de los coeficientes de actividad iónica de los cationes mayores en función de la fuerza iónica. A partir de estas gráficas podemos observar que se manifiestan de dos formas. El primer grupo, cuyos coeficientes de actividad disminuye hasta alcanzar un mínimo, para luego aumentar a medida que aumenta la fuerza iónica en la solución, son $\mathrm{yNa}^{+}, \mathrm{yMg}^{++}, \mathrm{yCa}^{++}, \mathrm{yCl}^{-}$. El segundo grupo lo constituyen los $\mathrm{yK}^{+}, \mathrm{ySO}_{4}^{--}, \mathrm{yHCO}_{3}^{-}$, que disminuye el valor de sus coeficientes de actividad, a medida que la solución se concentra (Figura 4 se representan con línea de tendencia).

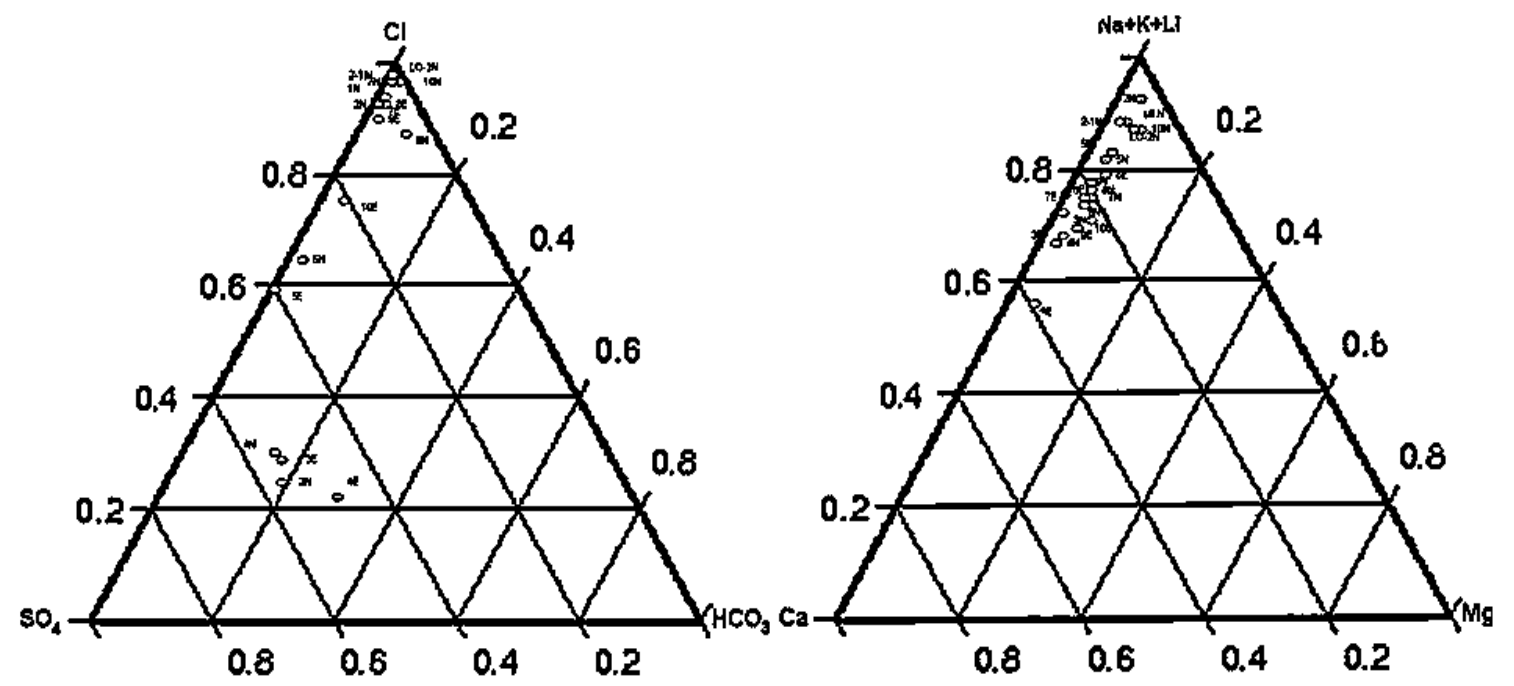

Fig.3: Representación gráfica en diagrama triangular de las aguas del salar de Loyoques.

$\mathrm{El} \mathrm{pH}$ que presentan estas aguas es ligeramente alcalino, oscilando sus valores extremos entre 7.2 y 9.02. La Tabla 2 muestra los resultados de los índices de saturación de los principales minerales posibles de precipitar. Estos muestran que las aguas se encuentran casi en situación de equilibrio con la calcita y aragonito, siendo el mineral siguiente más próximo al equilibrio el yeso, pero aún lejos del equilibrio, principalmente por las bajas concentraciones en la que se encuentran estas aguas $(0.004<\mathrm{F}$.I. $<3.65)$.

Los cálculos de equilibrio entre fases a partir de soluciones de electrolitos recobran importancia en el diseño y optimización de procesos de separación de sales a partir de salmueras naturales, siendo de interés industrial la aplicación de métodos de precipitación de sales a partir de sistemas multicomponentes. Estos muestran que todas las aguas están sobresaturadas en calcita (Fig. 5). El siguiente paso es considerar la alcalinidad (alc). Si la relación de concentración (alc) $>2\left(\mathrm{Ca}^{++}\right)$en el agua inicial del flujo de entrada, entonces aumentará regularmente durante el proceso evaporativo, siempre y cuando sólo precipite la calcita. En otras palabras, el producto de actividad de los iones $\mathrm{Ca}^{++}$en solución, en relación a los iones $\mathrm{CO}_{3}{ }^{-2}$ debe permanecer constante, a medida que el proceso de concentración aumenta. Si uno de los solutos aumenta, entonces el otro deberá disminuir. En nuestro caso, la distribución de toda el agua de entrada debe convertirse en salmueras ricas en carbonato. Sin embargo, la evolución del agua representada en el diagrama triangular (Fig. 3) es de tipo Na$\mathrm{Cl}-\mathrm{SO}_{4}$ con pH inferior a 9. La explicación se debe a que durante el proceso de evaporación ocurre la precipitación de Mg-silicatos (Hardie y Eugster, 1970). 


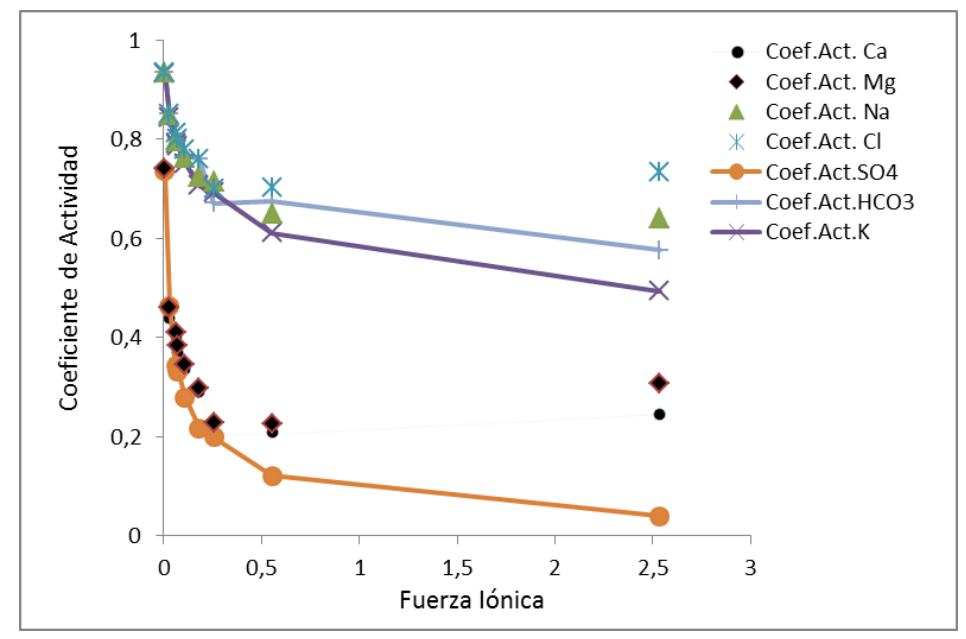

Fig. 4: Coeficientes de actividad iónicos en función de la fuerza iónica para aguas del Salar de Loyoques.

La Fig. 5 muestra las aguas saturadas en calcita y el yeso, dentro de cierto valor de incertidumbre del I.S. A partir de los datos termodinámicos propuestos por Greenberg y Moller (1989), se obtuvo el valor para la constante de equilibrio de la thernardita $\left(\mathrm{Na}_{2} \mathrm{SO}_{4}\right)$, valor no considerado en la base de datos original del código, siendo de interés para salmueras naturales con concentraciones más elevadas. Del mismo modo se han obtenido los valores, a distintas temperaturas, de las constantes de equilibrio de otras fases minerales de interés como la glauberita $\left(\mathrm{Na}_{2} \mathrm{Ca}\left(\mathrm{SO}_{4}\right)_{2}\right)$, que aparecían tabuladas en la base de datos original del código PHRQPITZ únicamente a $25^{\circ} \mathrm{C}$. La precisión alcanzada en el cálculo de los índices de saturación depende de la calidad tanto de los parámetros termodinámicos manejados (los incluidos en la base de datos del código y los procedentes de las necesarias ampliaciones, como la ya comentada de las constantes de equilibrio) como de los resultados analíticos obtenidos. El criterio aplicado para valorar la incertidumbre ha sido \pm 0.15 unidades de I.S., implicando que las situaciones de equilibrio no podrán determinarse con una precisión superior a la definida (Jenne, et al., 1980). En el caso de los minerales carbonatados se ha considerado un rango de incertidumbre algo superior, de \pm 0.4 unidades de I.S. Este tratamiento diferente se debe a la metodología para evaluar el pH en soluciones concentradas (Dickson, 1984; Markus, 1989), ya que el empleo de tampones convencionales para el calibrado del $\mathrm{pH}$-metro condiciona que la medida de este parámetro en salmueras no se encuentre en la misma escala de coeficientes de actividad que el modelo acuoso empleado (Plummer et al., 1988). Este tipo de problemas relativos al comportamiento en sistemas carbonatados a altas concentraciones, también se ha sido declarado en estudios de tipo experimental (Lazar et al., 1983). El código PHRQPITZ, es el más adecuado para el tratamiento de soluciones muy concentradas. Estudios similares se presentan en Monnin y Schott, 1984; Wen Qi y Mianping Zheng 2007, López et al, 1999; Garcés y López 2007, Garcés y López 2012.
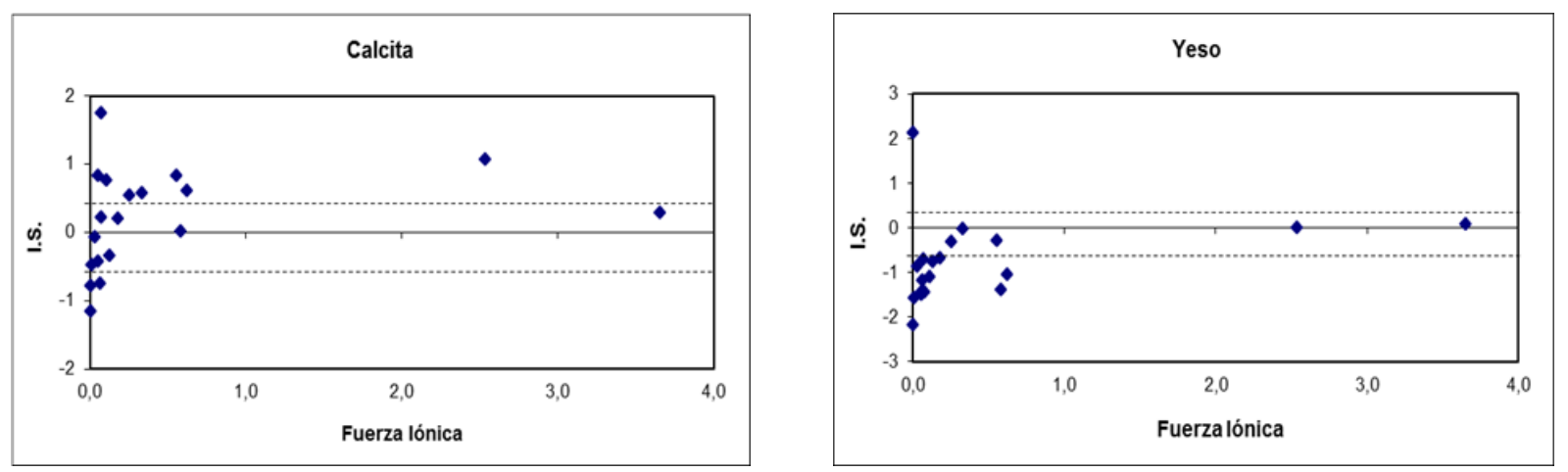

Fig. 5: Índices de Saturación de la calicita y yeso de las aguas del Salar de Loyoques

Estos resultados sugieren que la distribución de las aguas de entrada debe convertirse en alcalina, pero que en realidad son salmueras neutras. Esto induce a que las aguas evaporíticas siguen dos vías de evolución. Si durante el proceso evaporativo se enriquecen de carbonatos, llegando a ser una salmuera alcalina de carbonato de sodio, presentarán pH mayor a 10. La otra vía es cuando las aguas de aporte presentan más calcio que carbonato, de tal forma que la solución se concentra en calcio produciendo salmueras neutras con $\mathrm{pH}$ menor a 9, como lo es en nuestro caso. Por lo tanto, la relación fundamental si (alc) $>2\left(\left(\mathrm{Ca}^{++}\right)+2\left(\mathrm{Mg}^{++}\right)\right)$ no depende de la naturaleza del anión débil - ácido asociado a $\mathrm{Ca}$ y $\mathrm{Mg}$ durante la concentración por evaporación. Otra explicación de los datos es la existencia de un exceso de sulfato de origen. En este modelo, el azufre adicional se pone en las aguas del acuífero donde se oxida a sulfato y elimina los minerales de 
sulfato. Si todo catión alcalino se elimina como sulfato de la cantidad de sulfato oxidado, sería la diferencia entre la pérdida total de cationes alcalinos y pérdida aparente de sulfato. De la observación en el área del salar, muy próximo existe azufre nativo, por lo que éste es transportado por los vientos. Estos procesos dan como resultado una reducción significativa de la alcalinidad.

En ambientes continentales áridos a semiáridos con volcanismo activo, los boratos son ejemplo de sedimentación química evaporítica, cuyo aporte proviene de fuentes termales ubicadas a lo largo de sistemas de fallas, por donde ingresan estas aguas. La representación de la sedimentación en dirección desde los bordes hacia el centro, se tienen carbonatos (simbolizado por travertinos termales o termo-lacustres), luego sulfatos (yeso), boratos (ulexita y/o bórax) y cloruros. En aquellos salares en que aumentan los contenidos de boratos y de boro en las salmueras, aumenta también el contenido de litio y potasio, al punto que los salares con sedimentación boratífera, son aquellos que presentan valores anómalos y hasta económicos de litio y potasio (Salar de Surire en Garcés y López, 2007), esto se refleja en las muestras Q5-E y Q2-1N, concordando con los salares argentinos Hombre Muerto, Pozuelos y Rincón (Alonso, 2012).

Tabla 2: Índices de Saturación de las fases minerales con mayor posibilidad de presentarse por precipitación de las aguas del salar de Loyoques.

\begin{tabular}{|l|c|c|c|c|c|}
\hline Muestra & I.S. anhidrita & I.S. aragonito & I.S. calcita & I.S. yeso & I.S. Magnesita \\
\hline Q1-1N & -0.53 & 0.63 & 0.83 & -0.29 & -0.29 \\
\hline Q1-2N & -0.16 & 0.87 & 1.06 & -0.01 & 0.22 \\
\hline Q2-1N & -0.09 & 0.07 & 0.27 & 0.07 & -0.59 \\
\hline Q3N & -1.83 & -0.69 & -0.50 & -1.57 & -1.88 \\
\hline Q4N & -2.38 & -1.35 & -1.17 & -2.60 & -2.60 \\
\hline Q5N & -0.29 & 0.37 & 0.57 & -0.03 & -0.30 \\
\hline Q6N & -1.65 & 1.55 & 1.74 & -1.43 & 0.77 \\
\hline Q7N & -1.73 & -1.00 & -0.81 & -1.48 & -2.06 \\
\hline Q8N & -1.69 & -0.69 & -0.44 & -1.45 & -1.78 \\
\hline Q9N & -1.03 & -0.55 & -0.36 & -0.77 & -1.68 \\
\hline Q10N & -1.29 & 0.41 & 0.60 & -1.05 & -0.05 \\
\hline Q3E & -1.07 & -0.28 & -0.09 & -0.85 & -1.49 \\
\hline Q4E & -2.38 & -0.98 & -0.79 & -2.13 & -2.32 \\
\hline Q5E & -0.52 & 0.34 & 0.52 & -0.32 & -0.11 \\
\hline Q6E & -1.26 & 0.56 & 0.74 & -1.10 & -0.24 \\
\hline Q7E & -1.37 & -0.95 & -0.76 & -1.19 & -2.03 \\
\hline Q8E & -1.59 & -0.19 & -0.002 & -1.39 & -1.30 \\
\hline Q9E & -0.92 & 0.01 & 0.19 & -0.68 & -1.15 \\
\hline Q10E & -0.93 & 0.01 & 0.20 & -0.70 & -0.98 \\
\hline
\end{tabular}

\section{CONCLUSIONES}

Las aguas superficiales del Salar de Loyoques poseen una variabilidad en su tipo químico, son levemente alcalinas y mayoritariamente corresponden al tipo $\mathrm{Na}-\mathrm{Cl}-\mathrm{SO}_{4}$. Estas están claramente influenciadas por los contenidos de boro y arsénico cuyo origen es volcánico. Las aguas muestran una considerable variedad tanto en la composición iónica, como en su concentración. Estas aguas ingresan a la cuenca que es cerrada, bajo un clima árido con volcanismo activo y se someten a procesos de concentración por evaporación. A través del formulismo de Pitzer se evaluaron los coeficientes de actividades, y se determinó que el primer mineral a precipitar de las aguas es la calcita y sólo algunas muestras están próximas al equilibrio con yeso y magnesita.

\section{AGRADECIMIENTOS}

La autora agradece a la U. de Antofagasta y al Centro de Información Tecnológica de La Serena-Chile, por la facilidad prestada para la realización del trabajo.

\section{REFERENCIAS}

Alonso, R.N., Icnitas de aves en depósitos de boratos y su contribución a la reconstrucción paleoambiental, Revue de Paléobiologie, Genève, Vol. spéc. ISSN: 1661-5468, 11, 429-445 (2012)

Auqué, L. F., V. Valles y otros tres autores, Geoquímica de las lagunas saladas de Los Monegros (Zaragoza). 1. Determinación experimental del reequilibrio mirabilita-solución con la temperatura en un sistema natural, Estudios Geol., 51, 243-257 (1995) 
Ball, J.W. y D.K. Nordstrom, User's manual for WATEQ4F, with revised thermodynamic data base and test cases for calculating speciation of major, trace, and redox elements in natural waters: U.S., Geological Survey Open-File Report 91-183,189 p. (1991)

D.G.A., Balance Hidrológico Nacional, Dirección General de Aguas, DGA, Ministerio de Obras Públicas, Transporte y Telecomunicaciones, Gobierno de Chile, MOP. 24 p. (1987)

Dickson, A.G., pH scales and proton-transfer reactions in saline media such as sea water, Geochimica et Cosmochimica Acta, 48, 2299-2308 (1984)

Garcés, I. y P. López, Antecedentes Hidroquímicos del Salar de Aguas Calientes I (Chile), Rev. Fac. Ing. Univ. Antioquia, 62, 91-102 (2012)

Garcés, I. y P. López, Origin of borate deposit of Salar de Surire saline system, Acta con comité editor, $4^{\text {th }}$ International Limnogeology Congress (ILIC2007), Barcelona, España (2007)

Greenberg, J. P. y N. Moller, The prediction of mineral solubilities in natural waters: A chemical equilibrium model for the NaK-Ca-Cl'SO $-\mathrm{H}_{2} \mathrm{O}$ system to high concentration from 0 to $250^{\circ} \mathrm{C}$, Geochimica et Cosmochimica Acta, 53, $2503-2518$ (1989)

Hamrouni, B. y M. Dhahbi, Thermodynamic description of saline waters e prediction of scaling limits in desalination processes, Desalination, 137 (1-3), 275-284 (2001)

Hardie, L.A. y H.P. Eugster, The evolution of closed-basin brines, Mineralogical Society of America Special Paper, 3, 273-290 (1970)

Harvie, C.E., N. Moller y J.H. Weare, The prediction of mineral solubilities in natural waters: The Na-K-Mg-Ca- $\mathrm{H}-\mathrm{Cl}-\mathrm{SO} 4-\mathrm{OH}-$ $\mathrm{HCO}_{3}-\mathrm{CO}_{3}-\mathrm{CO}_{2}-\mathrm{H}_{2} \mathrm{O}$ system to high ionic strengths at 25으, Geochimica et Cosmochimica Acta, 48 (4), $723-751$ (1984)

Instituto Nacional de Normalización. NCh 1.333.Of78 Modificada 1987. Requisitos de Calidad del Agua para Diferentes Usos, Santiago, Chile 15p (1978)

Jenne, E. A., J. W. Ball, J. M. Burchard, D. V. Vivit y J. H. Barks, Geochemical modeling: apparent solubility controls on $\mathrm{Ba}, \mathrm{Zn}, \mathrm{Cd}, \mathrm{Pb}$ and $\mathrm{F}$ in waters of the Missouri Tri-State mining area. Trace Substances in Environmental Health, 14, 353361. D. D. Hemphill (Editor), University of Missouri, Columbia, Montana (1980)

Krumgalz, B., Application of the Pitzer ion interaction model to natural hypersaline brines, J. of Molecular Liquids, 91 3-19 (2001)

Lazar, B., A. Starinski, A. Katz, E. Sass y S. Ben-Yaakov, The carbonate system in hypersaline solutions: alkalinity and $\mathrm{CaCO}_{3}$ solubility of evaporated seawater, Limnology and Oceanography, 28, 978-986 (1983)

López, P., L. Auque, I. Garcés y G. Chong, Características Geoquímicas y pautas de evolución de las salmueras del Salar de Llamará, Chile, Revista de la Sociedad Geológica de Chile, 26 (1), 89-108 (1999)

Markus, Y., Determination of pH in highly saline waters, Pure and Applied Chemistry, 61, 1133-1138 (1989)

Monnin, C. y J. Schott, Determination of the solubility products of sodium carbonate minerals and an application to trona deposition in Lake Magadi (Kenya), Geochimica et Cosmochimica Acta, 48(3), 571-581 (1984)

Peel, M.C., B.L. Finlayson y T.A. McMahon, Updated world map of the Köppen-Geiger climate classification, Hydrology and Earth System Sciences Discussions, 4(2), 439-473 (2007)

Pliscoff, P., F. Luebert, H. Hilger y A. Guisan. Effects of alternative sets of climatic predictors on species distribution models and associated estimates of extinction risk: A test with plants in an arid environment, Ecological Modelling, 288, 166-177 (2014)

Pitzer, K.S. y G. Mayorga, Thermodynamics of electrolytes. II. Activity and osmotic coefficients for strong electrolytes with one or both ions univalent, J. Phys. Chem., 77 (19), 2300-2308 (1973)

Pitzer, K.S. y J.J. Kim, Thermodynamics of electrolytes. IV. Activity and osmotic coefficients for mixed electrolytes, J. Am. Chem. Soc., 96 (18), 5701-5707 (1974)

Pitzer, K.S., Thermodynamics of electrolytes, V. Effects of higher-order electrostatic terms, J. Solut. Chem., 4 (3), $249-265$ (1975)

Pitzer, K., Activity coefficients in Electrolyte Solutions, Cap. 3, 2ª Ed., CRC Press, Boca Ratón. (1991)

Plummer, L.N., D. L. Parkhurst, G. W. Fleming y S.A. Dunkle, Computer program incorporating Pitzer's equations for calculation of geochemical reactions in brines, Water-Resources Investigation Report, WRI 88-4153 (1988)

Risacher, F., H. Alonso y C. Salazar, Geoquímica de aguas en cuencas cerradas: I, II Y III Regiones - Chile. Vol III. Estudio de cuencas de la II región. S.I.T. N 51. Convenio de Cooperación DGA - UCN - IRD. [LOY].121 - [LOY] 133 (1999)

Sarricolea, P., M.J. Herrera-Ossandón y O. Meseguer-Ruiz, Climatic regionalization of continental Chile, J. Maps, 13(2), 66-73 (2017)

Weare, J.H., Models of mineral solubility in concentrated brines with application to field observations. En: Thermodynamic Modeling of Geochemical Materials Minerals, Fluids and Melts, 17, 143-176, Reviews in Mineralogy (1. S. E. Carmichael, y H. P. Eugster, Edits.), Mineralogical Society of America (1987)

Wen Qi. y Mianping Zheng, Simulation with Pitzer model of lake brine evolution of Zabuye salt lake, Tibetan plateau, Acta Geologica Sinica 81, (12), 1734-1741 (2007) 